\title{
Making the Stitching Process of Montaged SEM Images Automatic Using Fourier Transform Properties
}

Nasim Khoonkari ${ }^{1}$, Christopher Anand ${ }^{2}$ and Nabil Bassim ${ }^{3}$

${ }^{1}$ Computing and Software Department, McMaster University, Hamilton, Ontario, Canada, Hamilton, Ontario, Canada, ${ }^{2}$ Computing and Software Department, McMaster University, Hamilton, Ontario, Canada, Canada, ${ }^{3}$ Department of Materials Science and Engineering, McMaster University, Hamilton, Ontario, Canada, Hamilton, Ontario, Canada

Acquiring several images of parts of a sample at high magnification and assembling them into a single composite image is called montaging, which can be done either by moving the stage in an X-Y raster then capturing snapshots from an SEM or by using a unique tool like a Multi-SEM [4]. Usually, a degree of overlap is preserved between adjacent images, or tiles, which is used to align these images along vertical and horizontal seams, a process is known as stitching.

The automatic creation of a large high-resolution montaged image is a growing research area involving computer vision and image processing. Achieving the stitched image is time-consuming and inefficient manually; there are some attempts at automating this process like the MIST image stitching tool [2]. This study attempts to address the image stitching problem for two cases in the montaging of semiconductor metallization layers for microelectronics applications.

Intentional shift, where one slides one tile on the overlap area of the other tile to resolve the shift and stitch the images from the overlap area; Instrument drift, which is usually a change in the imaging conditions related to a change in temperature in the laboratory.

Instrument drift happens during the scan and is not predictable. We deployed a Fourier transform-based method to determine the degree of the shift caused by the instrument. The shift theorem states that delaying a signal $x(t)$ by 'a' seconds multiplies its Fourier transform by e-jwa [1]. We chose a part of the image with the obvious instrument drift and summed over the columns and rows of the regions of interest in the images separately. Therefore, we could take advantage of 1D Fourier transform rather than the 2D, which facilitates a faster calculation and is temporally in sync with the $\mathrm{X}-\mathrm{Y}$ raster mechanics of the scan generation pattern in the SEM. Multiplication of the Fourier transform of the resulting line from the first image by the conjugate of Fourier transform of its shifted one will return the shift's amount (signal delay). We put a window, 'Lanczos window', on the image to reduce ringing artifacts. Mathematically, outside of the window interval is having a zero value when a wave is multiplied by a window function. So, all that is left is the part where they overlap, which is called the 'view through the window'. The isolated segment of the data within the window is multiplied by the window function values called tapering. Lanczos window is the normalized sinc function sinc $(\mathrm{x})$, which is the central lobe of a horizontally stretched sinc function $\operatorname{sinc}(x / a)$ for $-a \pi \leq x \leq a \pi$, see Fig 1c. In particular, there may be ringing artifacts just before and after abrupt changes in the sample values, which may lead to clipping artifacts. Given the special role of edge sharpness in vision, these effects are reduced, and the image's subjective quality is improved by using the sinc filter. So, we formulated our problem as,

FFT (Lineimg1 $\{\mathrm{L} \cdot \mathrm{img} 1\}) \cdot(\mathrm{FFT}($ Lineimg2 $\{\mathrm{L} \cdot \mathrm{img} 2\}) *$

where '*' means conjugate, Lineimg1 is a row-wise or columns-wise summation of the sub-image of the Img1 and Lineimg2 is the same for Img2, and 'L' stands for Lanczos window (in order to adjust the window, we used a square root of it).

Figure 1a is an example of two SEM images having 500 pixels overlap along the horizontal seam at the top of the image (highlighted in red). These images are taken using a scanning electron microscope (SEM) 
with backscattered electrons. We tried our algorithm on parts of the image with some landmarks to detect the shift better because in plain areas with repeated patterns, the shift is more difficult to estimate accurately, see Fig $1 b$.

The result of applying the Lanczos window on the image is shown in Fig 1c. We summed up the result both column-wise and row-wise to get two vectors which can be used as inputs for the Fourier shift theorem.

The Fourier transform is a complex-valued function of frequency. Complex numbers can be represented in Cartesian or Polar coordinates. A complex number $z=\operatorname{Re}(z)+i \operatorname{Im}(z)$ in Cartesian coordinates can be shown by its 'Phase' and 'Magnitude' as $\mathrm{z}=\operatorname{rei} \Theta$, where $\mathrm{r}=|\mathrm{z}|$ is the magnitude and $\Theta=\operatorname{atan} 2(\operatorname{Im}(\mathrm{z}), \operatorname{Re}(\mathrm{z}))$ is the phase. By calculating the angle using 'atan2' and number of phase changes by $\arctan \times$ length$($ Line $)) /(2 \times \pi)$, one can calculate the number of phase changes over time, which is 19.13 for rows and 47.95 in columns. These amounts match the shift we detected in the first step by plotting the lines we got from summing the rows and columns of the regions of interest in the images, see Fig 2. One advantage of using this method is that we can calculate even fractional shifts.

When this method is compared to other available methods like cross-correlation, Entropy, Mutual Information, and Sum of Square differences [3], we find this method is better in calculating even a subpixel shift. This algorithm can calculate the instrument drift, one of the most important problems in image stitching.

(a)
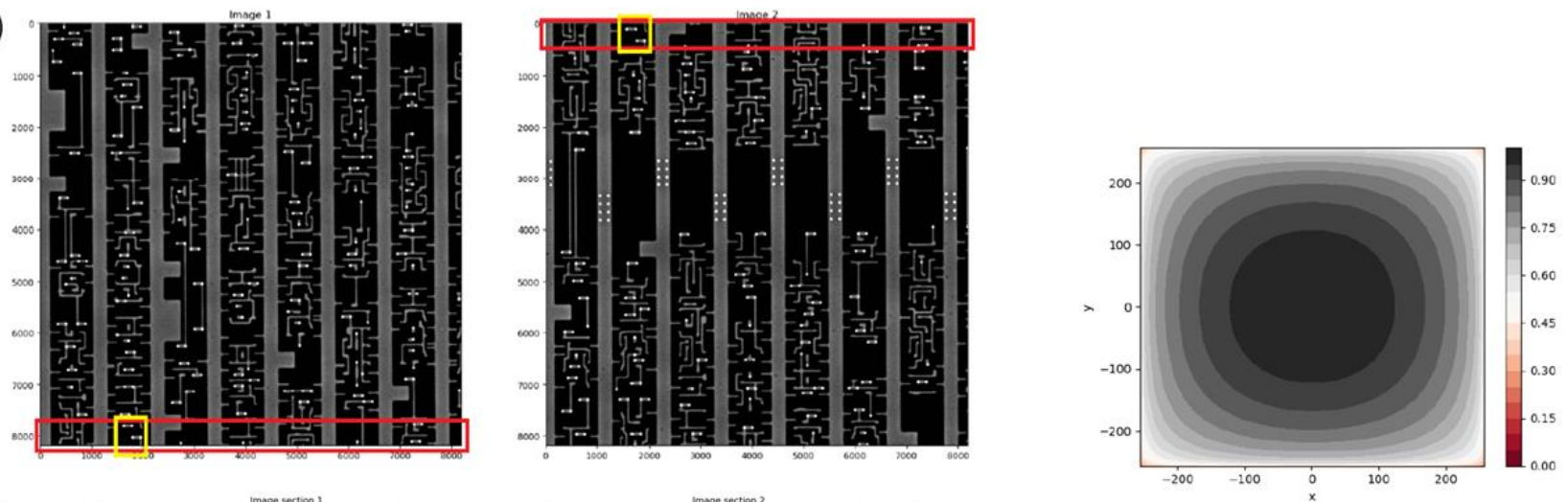

(b)
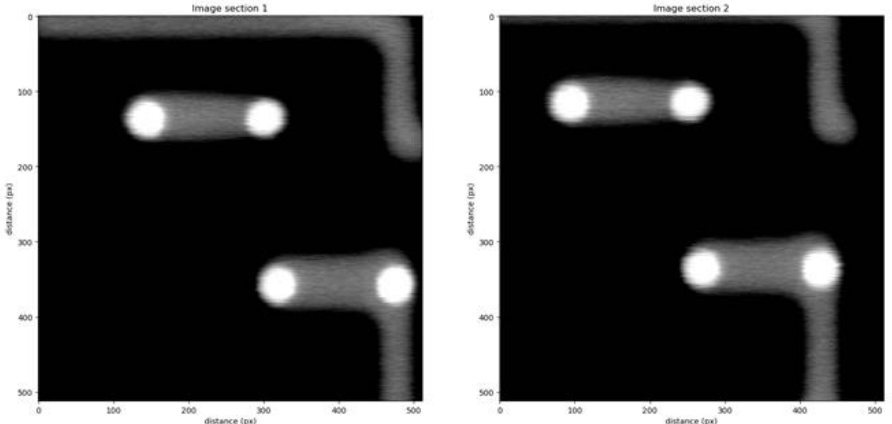
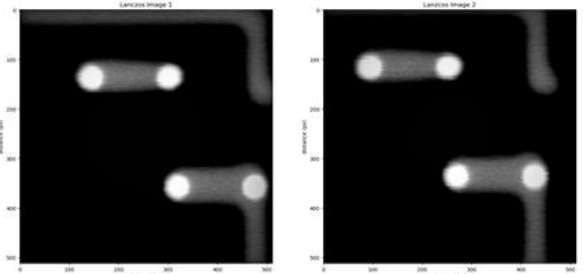

(c)

Figure 1. (a) Representative BSE SEM images that are part of a larger montage experimental run from a semiconductor metallization sample (magnification: $7.743959 \mathrm{~mm}$ ). The red box denotes a 500-pixel overlapping region between the two images. The yellow box is a sub-tile we chose to work on from the overlap area. (b) A subtile from the overlap, which is marked as yellow in (a). (c) top: Square root of the Lanczos window; reduce the artifacts of the image (The spurious signals near the sharp transitions in signals are called ringing artifacts which appear as bands or ghosts near edges), bottom: The image subtiles are multiplied by the Lanczos window. 

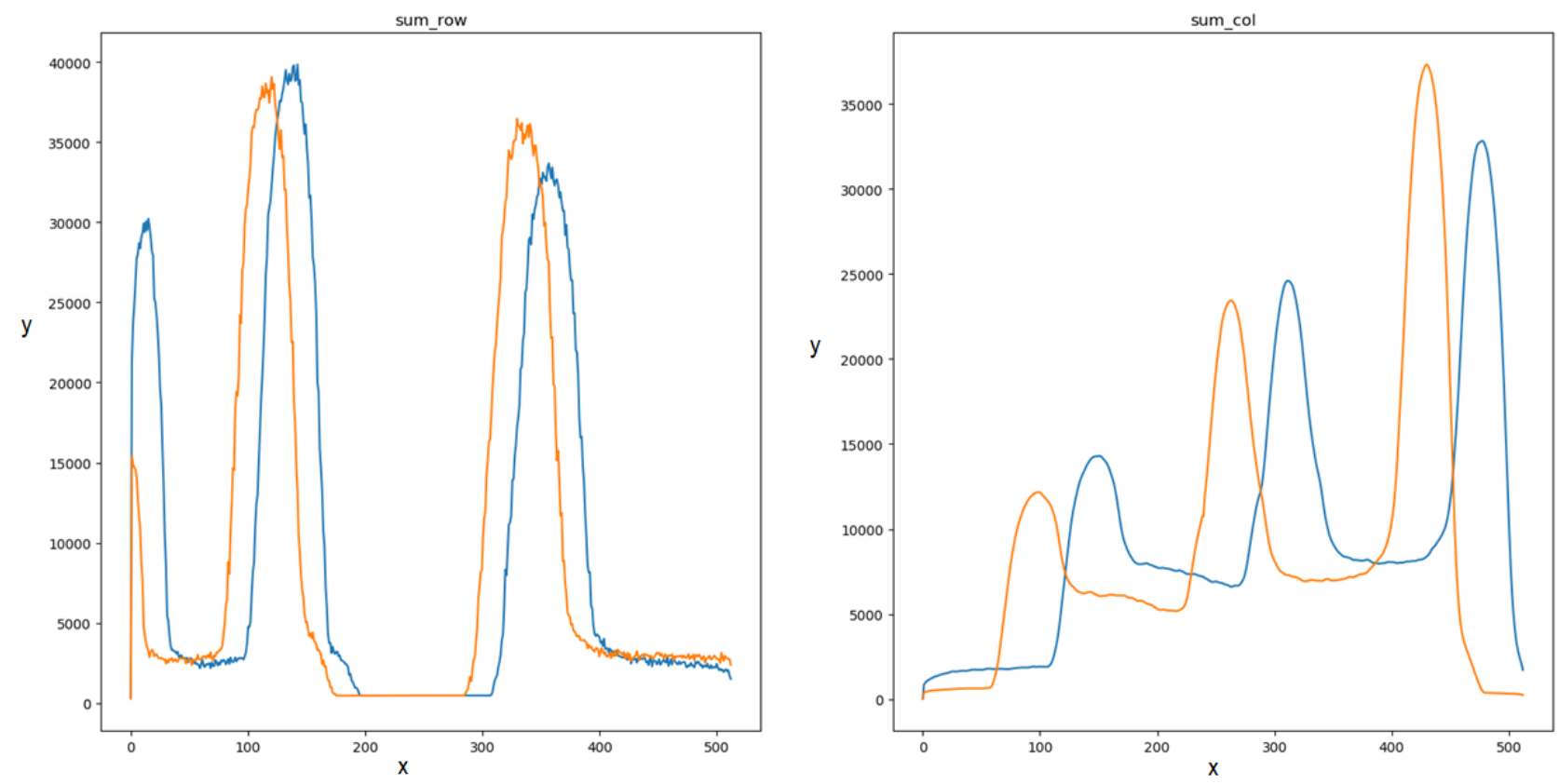

Figure 2. Plot of the lines obtained from row-wise and columns-wise summation of the regions of interest in the images. The result of the orange line is from Img1 and the blue line is from Img2. Left plot is for row-wise summation and right one stands for column-wise summation. The result from calculation 'arctan $\times$ length $($ Line $)) /(2 \times \pi)$ ' matches this graph as expected, showing a shift of 47.95 pixels in the $x-$ direction and 19.13 pixels in the $\mathrm{y}$-direction. $\mathrm{X}$-axis is for the pixels of the line obtained from the rowwise and column-wise summation and Y-axis is for the summation values.

\section{References}

[1] Bracewell, Ronald Newbold and Bracewell, Ronald N The Fourier transform and its applications, McGraw-Hill New York, vol 31999, 1986.

[2] Chalfoun, Joe and Majurski, Michael and Blattner, Tim and Bhadriraju, Kiran and Keyrouz, Walid and Bajcsy, Peter and Brady, Mary Mist: accurate and scalable microscopy image stitching tool with stage modeling and error minimization, Scientific reports, Nature Publishing Group, vol 7(1), 2017.

[3] Mahalakshmi, T and Muthaiah, R and Swaminathan, P Image Processing, Research Journal of Applied Sciences, Engineering and Technology, vol 4(24), 2012.

[4] Eberle, Anna Lena and Garbowski, Tomasz Large-Scale Automated Serial Section Imaging with a Multibeam Scanning Electron Microscope, Springer, vol Microscopy, 2020. 